\title{
Biosimilars in IBD: Similarity Breeds Contented Patients
}

\author{
Vito Annese ${ }^{1}$ \\ Published online: 6 February 2019 \\ (c) Springer Science+Business Media, LLC, part of Springer Nature 2019
}

The introduction of biologic therapy for the treatment of inflammatory bowel disease (IBD) during the last two decades represents a considerable breakthrough in the management of these patients with positive impacts on quality of life and the need for hospitalization and surgery [1]. Despite these advantages, the progressive increase in medication expenditures has burdened the global healthcare budget, limiting the access to biologic therapies to many patients. In IBD specifically, biologic agents are primary drivers of budgetary increases for overall care [2].

As the patent of infliximab (IFX) and other biologic agents has or soon will expire, an inevitable consequence is the emergence, development, and use of biosimilars, defined as therapeutic antibodies closely resembling but not identical to the original product (originator). Their introduction generates competition in the pharmaceutical market and reduces the economic burden of therapy, thus providing enhanced access to biologic therapies and in general saving healthcare resources $[3,4]$.

CT-P13 (Celltrion, Inc. Incheon, Republic of Korea) was the first biosimilar monoclonal antibody of infliximab approved by the European Medicines Agency (EMA) and the US Food and Drug Administration (FDA) that has been marketed since 2013. Despite its availability, the use of biosimilars has been debated due in part to the regulatory process governing these antibodies. In contrast to the reference product pathway, wherein the proof of efficacy is needed for each disease, the use of biosimilars is extended to all the other indications for reference product, without the need of specific clinical trials [5]. Moreover, it is still unclear whether switching from the originator to biosimilars only for economic reasons is appropriate in a patient with good disease control, due to the possible occurrence of a nocebo effect (negative perceived effects of a different medication) or due to true inferiority of the biosimilar. In this regard,

Vito Annese

vito.annese@valiant.ae

1 Valiant Clinic \& American Hospital, Dubai, UAE only a single randomized controlled noninferiority, doubleblinded, phase 4 trial with 52 weeks of follow-up is available [6] enrolling 482 patients with different immune-mediated diseases, including 155 patients with Crohn's disease (CD). After switching, a nonsignificant trend toward more frequent disease worsening was recorded in CD patients using CT-P13 compared to those continuing IFX therapy (36.5\% vs $21.2 \%$ ).

In this issue of Digestive Diseases and Sciences, Plevis et al. [7] report their experience with biologic drugs in a prospective evaluation of a single-center Scottish cohort of CD patients. The decision to switch from IFX to CT-P13 was discussed with the subjects within a specific information pathway, reassuring them that close follow-up, including IFX trough and antibody (ATI) levels, obtained before and after switching, would be provided to detect potential pharmacodynamic deviations. A total of $155 \mathrm{CD}$ patients receiving maintenance therapy with IFX were available to switch, and remarkably, all accepted. Following evaluation with therapeutic drug monitoring (TDM), CT-P13 was administered at an increased dose in 12 patients, while in 6 , the dose was reduced. Moreover, 21 patients were switched to adalimumab, and 24 patients eventually stopped the biologic therapy as they had achieved stable remission. Therefore, 110 patients received CT-P13 therapy who were followed during 756 infusions for $>104$ patient-years of followup. No significant difference of disease and inflammatory markers such as the Harvey-Bradshaw Index, C-reactive protein, and fecal calprotectin, or of IFX trough levels was observed when comparing data obtained before and at 6 and 12 months after switch. No infusion reactions or unexpected safety signals were reported. The authors also concluded that the average cost saving was roughly $50 \%$ compared with the cost of the originator which was $\sim \$ 800,000$ USD.

This study, although uncontrolled and not randomized, with limited sample size and follow-up, has a number of strengths: Firstly, it demonstrates a great confidence of patients with their doctors regarding the decision about switching that was assuredly related to an appropriate communication pathway; secondly, it supports the contention 
that part of the resources saved by using biosimilars can eventually be re-invested for the same patients by applying more widely a cost-effective TDM-based management. Besides the demonstration of the efficacy and safety of CT-P13, already reported in a number of other real-world experiences $[8,9]$, this study adds another important piece of information for patients and doctors that face this decision. Indeed, following TDM evaluation, therapy was modified either by increasing or reducing the dose or switching to another anti-TNF- $\alpha$ agent in about one-third of patients in stable clinical remission under IFX. Although the debate about having a proactive or reactive TDM evaluation is still ongoing [10], this study suggests a potential benefit of proactive management, although the study was not specifically designed to examine such an outcome.

A number of issues about the use of biosimilars in IBD remain to be evaluated, the most important being the safety of multiple switches between the reference product and biosimilar, and more importantly, among different biosimilars of the same reference product. If the basis of the choice to switch and eventually to switch again is principally economic, this may enter standard clinical practice given the number of biosimilars already available and under development, and is probably already a viable option for patients under treatment that move to different countries.

\section{Key Messages}

- Biosimilars will gradually replace older biologicals, with increasing use among IBD patients

- The use of biosimilars increases access to the most effect IBD treatments

- Ongoing professional and patient education is essential in order to establish patient confidence, especially when switching

- Cost saving may already be re-invested in patients using biosimilars into TDM and other beneficial interventions, increasing patient acceptance

- The safety and efficacy of CT-P13 have been largely demonstrated in IBD thanks especially to real-world experience studies such as that by Plevris N. et al.

- The issue of multiple switching deserves appropriate investigation and caution since it is preliminary and untested

\section{References}

1. Danese S, Vuitton L, Peyrin-Biroulet L. Biologic agents for IBD: practical insights. Nat Rev Gastroenterol Hepatol. 2015;12:4-537.

2. Annese V, Duricova D, Gower-Rousseau C, Jess T, Langholz E. Impact of new treatments on hospitalisation, surgery, infection, and mortality in IBD: a focus paper by the epidemiology committee of ECCO. J Crohns Colitis. 2016;10:216-225.

3. Zheng MK, Shih DQ, Chen GC. Insights on the use of biosimilars in the treatment of inflammatory bowel disease. World J Gastroenterol. 2017;23:1932-1943.

4. 2017 IMS Biosimilar report-the impact of biosimilar competition in Europe. http://ec.europa.eu/growth/content/impac t-biosimilar-competition-price-volume-and-market-share-updat e-2017-0_en. 2 Jan 2019, data last access.

5. Feagan BG, Choquette D, Ghosh S, et al. The challenge of indication extrapolation for infliximab biosimilars. Biologicals. 2014;42:177-183.

6. Jørgensen KK, Olsen IC, Goll GL, et al. Switching from originator infliximab to biosimilar CT-P13 compared with maintained treatment with originator infliximab (NOR-SWITCH): a 52-week, randomised, double-blind, non-inferiority trial. Lancet. 2017;389:2304-2316.

7. Plevris N, Jones GR, Jenkinson PJ, Lyons M, Chuah CS, Merchant LM, et al. Implementation of CT-P13 via a managed switch programme in Crohn's disease: 12 month real-world outcomes. Dig Dis Sci. (Epub ahead of print). https://doi.org/10.1007/s1062 0-018-5406-8.

8. Meyer A, Rudant J, Drouin J, Weill A, Carbonnel F, Coste J. Effectiveness and safety of reference infliximab and biosimilar in Crohn disease: a French equivalence study. Ann Intern Med. 2019;170:99-107.

9. Bakalos G, Zintzaras E. Drug discontinuation in studies including a switch from an originator to a biosimilar monoclonal antibody: a systematic literature review. Clin Ther. 2019;41:155-173.

10. Vande Casteele N, Herfarth H, Katz J, Falck-Ytter Y, Singh S. American gastroenterological association institute technical review on the role of therapeutic drug monitoring in the management of inflammatory bowel diseases. Gastroenterology. 2017;153:835-857.

Publisher's Note Springer Nature remains neutral with regard to jurisdictional claims in published maps and institutional affiliations. 